\title{
Dislocation Dissociation and Short-Range Ordering in ZrN
}

\author{
P. Li and J.M. Howe
}

Department of Materials Science and Engineering, University of Virginia, Charlottesville, VA, 22904-4745, USA

Transition metal carbides and nitrides generally have a high stacking-fault energy (SFE), due to the bonding in these alloys. This leads to a narrow separation of dissociated dislocations so that direct observation of dislocation dissociation is difficult. Dissociation of $\{111\}\langle 110\rangle$ dislocations into Shockley partials in TiC has been reported by Tsurekawa and Yoshinaga [1], and estimates of the SFE range from 130 to $300 \mathrm{~mJ} / \mathrm{m}^{2}$. The present research was undertaken to determine whether or not dislocation dissociation occurs in $\mathrm{ZrN}$ alloy. Sheets of $\mathrm{ZrN}$ alloy were annealed at $1200^{\circ} \mathrm{C}$ and quenched in room-temperature water. Transmission electron microscopy (TEM) was performed on thin foils using a JEOL 2000FXII TEM at 200kV.

As shown in Fig. 1, it was found that perfect $1 / 2<110>$ dislocations dissociate into two $1 / 6<112>$ Shockley partial dislocations (arrows) bounding an intrinsic SF in $\mathrm{ZrN}$. This dissociation reaction is the same as that observed in usual FCC alloys and it has not been previously reported for transition metal nitrides with the $\mathrm{NaCl}$ prototype structure. All of the dislocations and SF's in $\mathrm{ZrN}$ were found to lie on the $\{111\}$ planes, indicating that a possible slip system is $\{111\}\langle 1 \overline{1} 0\rangle$. Trace analysis showed that the $1 / 2<110\rangle$ dislocations contain super-jogs that are not coplanar with the dissociated Shockley partials. The wide separation of the partial dislocations bounding the SF's indicates that the $\mathrm{SFE}$ in $\mathrm{ZrN}$ alloy is low, with a value of approximately $6 \mathrm{~mJ} / \mathrm{m}^{2}$. This unusually low SFE can be explained on the basis of a high vacancy concentration in $\mathrm{ZrN}$. Further dissociation of the Shockley partials does not occur, in contrast to the analysis of Kelly and Rowcliffe [2].

The high vacancy concentration in $\mathrm{ZrN}$ was confirmed by the appearance of diffuse intensity maxima in electron diffraction patterns, as shown in Fig. 2. Such diffuse intensity comes from the short-range ordering (SRO) of vacancies and nitrogen atoms. When a compound has many point defects, but an insufficient concentration to give long-range ordering, SRO may occur. This ordering causes diffraction effects and a clear example of this phenomenon occurs in metal carbides such as $\mathrm{TiC}$, and VC. In nitrides, evidence for diffuse intensity was found in TiN, but not in VN [3]. Diffuse intensity maxima have not been previously observed in $\mathrm{ZrN}$.

The shapes of the diffuse intensity maxima in $\mathrm{ZrN}$ are the same as those found in transition metal carbides. Sauvage and Parthé [4] have analyzed these diffuse intensity maxima theoretically. However, there is a significant increase the diffuse intensity at the $\{1,1 / 2,0\}$ positions in $\mathrm{ZrN}$ (arrows in Fig. 2(a)) as compared to transition metal carbides. Such diffuse intensity maxima at the $\{1,1 / 2,0\}$ positions have not been previously observed in binary metal carbides and nitrides with the $\mathrm{NaCl}$ prototype structure. Similar diffuse intensity maxima were found in TiMoC and these were explained by $\mathrm{Ti}_{4} \mathrm{Mo}$ metal-atom ordering, but this is different from $\mathrm{ZrN}$ where there is only one metal atom in the phase. Further investigation on the origin of the $\mathrm{SRO}$ in $\mathrm{ZrN}$ by in-situ irradiation in the TEM is currently under investigation [5]. 


\section{References}

[1] S. Tsurekawa and H. Yoshinaga, J. Japan Inst. Metals 58 (1994) 390.

[2] A. Kelly and D. J. Rowcliffe, Phys. Stat. Sol. 14 (1966) K29.

[3] J. Billingham et al., Acta Cryst. 28A (1972) 602.

[4] M. Sauvage and E. Parthé, Acta Cryst. 28A (1972) 607.

[5] This research was supported by NSF under grant DMR-9908855.
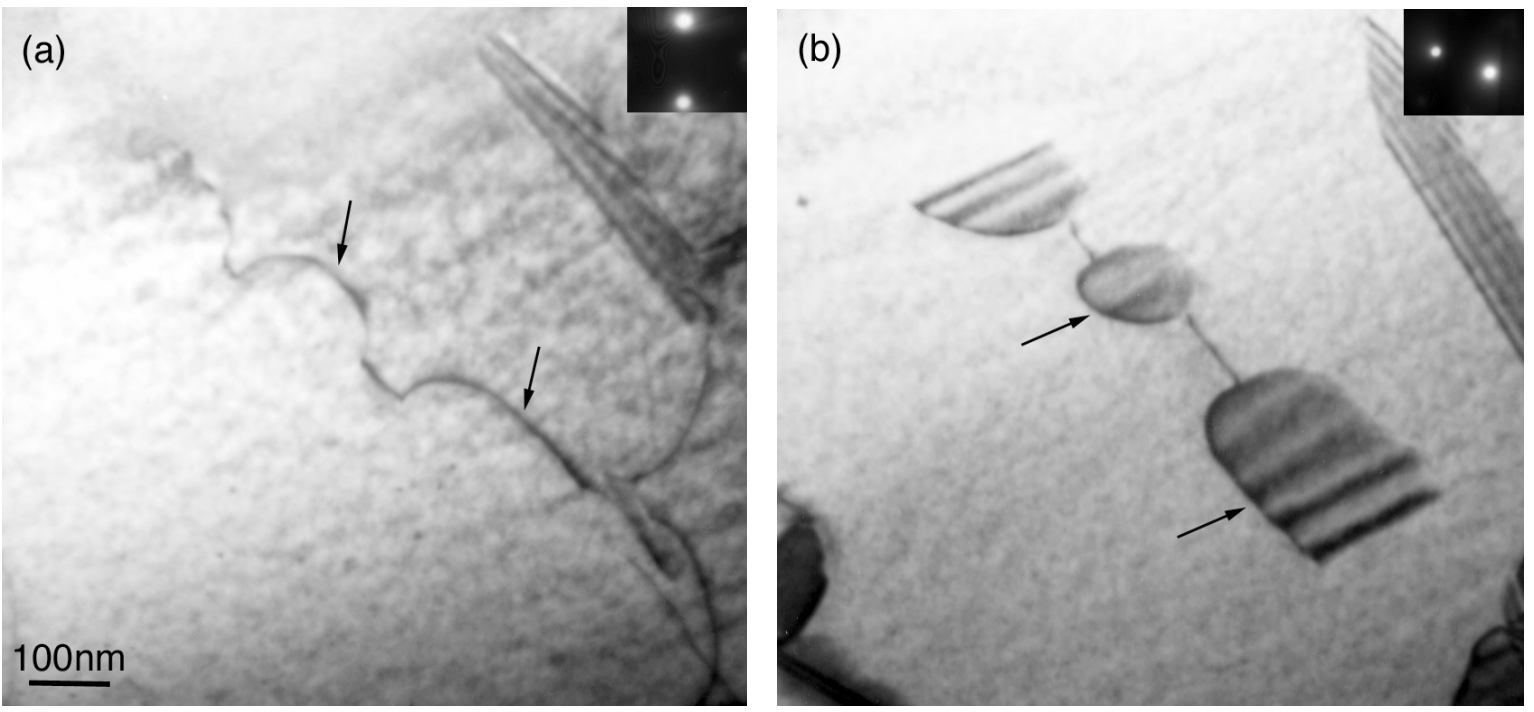

FIG. 1. Bright-field TEM images of dissociated dislocations under different two-beam diffracting conditions: (a) $\mathbf{g}=2 \overline{2} 0, \mathbf{B}=[111], \mathbf{b}=1 / 6[1 \overline{2} 1]$ visible (arrows), and (b) $\mathbf{g}=002, \mathbf{B}=[100]$, $\mathbf{b}=1 / 6[\overline{1} 12]$ visible (arrows).
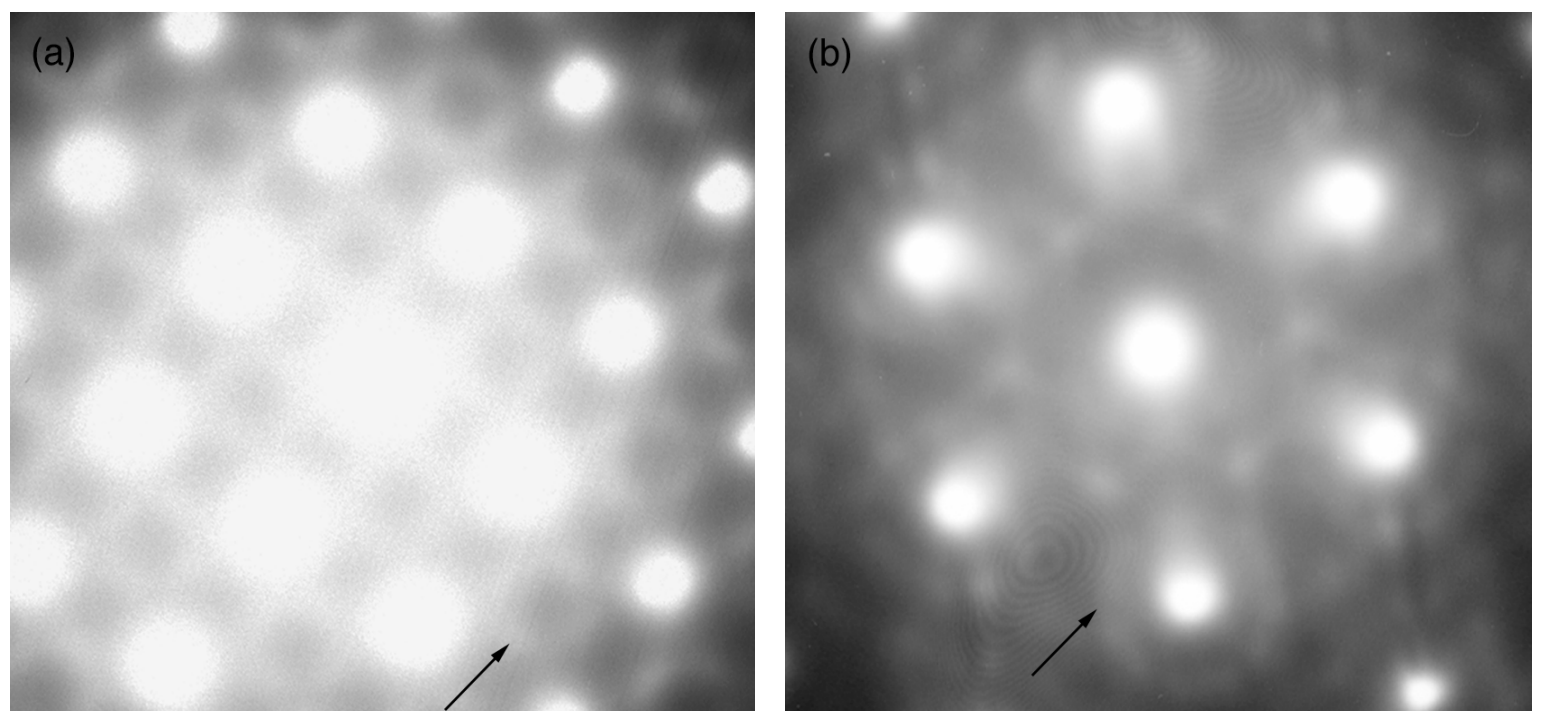

FIG. 2. Diffuse intensity maxima (arrows) in electron diffraction patterns from $\mathrm{ZrN}$ observed in two different zone axes due to SRO of vacancies: (a) $\mathbf{B}=[100]$, and (b) $\mathbf{B}=[111]$. 